\title{
Machine and Deep Learning Applications in Particle Physics
}

\author{
Dimitri Bourilkov \\ Physics Department, University of Florida, PO Box 118440 \\ Gainesville, Florida 32611, USA \\ dimi@ufl.edu
}

Received 25 October 2019

\begin{abstract}
The many ways in which machine and deep learning are transforming the analysis and simulation of data in particle physics are reviewed. The main methods based on boosted decision trees and various types of neural networks are introduced, and cutting-edge applications in the experimental and theoretical/phenomenological domains are highlighted. After describing the challenges in the application of these novel analysis techniques, the review concludes by discussing the interactions between physics and machine learning as a two-way street enriching both disciplines and helping to meet the present and future challenges of data-intensive science at the energy and intensity frontiers.
\end{abstract}

Keywords: Particle physics; Collider; Machine learning.

PACS numbers: 07.05.Mh

\section{Introduction}

The twenty-first century has brought widespread advances in the natural and social sciences by making them data-intensive. The rise in computing power and networking has allowed to amass ever expanding collections of data in the petabyte and even exabyte range ${ }^{2}$. The progress in social media and e-commerce has only added to the flood. This in turn has accelerated the development of novel techniques needed to analyze the data and extract useful and timely information from it. The field of data science was born.

The traditional way to analyze, or generate simulated, data is to first develop algorithms based on domain knowledge, then implement them in software, and use the resulting programs to analyze or generate data. This process is labor intensive, and analyzing complex datasets with many input variables becomes increasingly difficult and sometimes intractable. Artificial intelligence (AI) and the subfield of machine learning (ML) attack these problems in a different way: instead of humans

${ }^{\text {a }}$ For pioneering developments in 2001-5 see e.g. the International Virtual-Data Grid Laboratory for Data Intensive Science (iVDGL), combining the efforts of the Laser Interferometer Gravitationalwave Observatory (LIGO), the ATLAS and CMS detectors at LHC at CERN and the Sloan Digital Sky Survey (SDSS)! 
developing highly specialized algorithms, computers learn from data how to analyze complex data and produce the desired results. There is no need to explicitly program the computers. Instead, ML algorithms use (often large amounts of) data to build models with relatively small human intervention. These models can then be applied to predict the behavior of new, previously unseen data, to detect anomalies or to generate simulated data. While early work stretches back more than fifty years, progress was slow for long periods of time. Advances in academic research paired with the needs of large companies like Google, IBM, Amazon, Facebook and Netflix, just to name a few, are producing a fundamental paradigm shift, especially with the recent successes of deep learning (for an excellent introduction to the topic, see e.g. $\left.{ }^{2}\right)$.

Using mostly traditional analysis methods, physics has advanced rapidly, establishing the Standard Model (SM) of particle physics, and more recently its cosmological homologue, $\Lambda \mathrm{CDM}$. The coming years will bring unprecedented amounts of data and complexity at the Large Hadron Collider (LHC), accelerating protons at CERN, as well as at the intensity frontier and elsewhere. Extracting the underlying physics in the same way becomes more and more challenging, or simply impossible in a timely manner. That explains the recent spark of interest in ML (for excellent recent reviews and plans for the future, see e.g. ${ }^{3 / 5}$ ).

The physical sciences are in a unique position. While in many other fields there are less firm theoretical foundations or models, physicists have well established methods to predict and to compare the results of experiments to theoretical calculations, as the many successes of the SM attest. This means that physics motivated ML methods can be developed and applied, accelerating the learning process and making it more efficient and precise. At the same time the breath-taking advances in data science and computing technology will help to address the coming challenges in particle physics.

This review is not meant to be all-encompassing. Rather, some cutting-edge applications at the energy and intensity frontiers of particle physics are selected to illustrate the many amazing ways in which ML is applied, and to highlight both the successes and the challenges. The review is organized as follows: after an introduction to ML, the applications in experimental high energy physics (HEP) are reviewed in section 2, and in phenomenological and theoretical HEP in section 3. Open issues and challenges are discussed in section 4, followed by a more general overview of how ML works or can be improved in section 5, and an outlook in section 6 .

\subsection{Machine Learning Basics and Vocabulary}

With the increasing complexity of events in high energy physics, the importance of multivariate analysis for LHC has been recognized before the start of data taking. The main motivation was to go beyond the traditional methods for event selection 
by applying series of cuts on individual variables, and be able to use correlations and more intricate patterns in the multidimensional data. A workshop ${ }^{6}$ at Caltech in 2008 was dedicated to the topic; ML techniques were practically not on the radar. What a sea change ten years later.

Machine learning algorithms, which are general in nature and not task-specific, are geared towards improving the measurable performance on some given task by training on more and more data. The data are split in training, validation and test subsets. The first two are often combined together, as in cross-validation, where a different chunk of the data is used at each training step to estimate the predictive power of a model. The ultimate measure of the model generalization ability is how it will perform on unseen test data, which can include real or future data. To avoid the danger of overfitting, in ML approximate solutions are preferred: the goal is to learn the essential features of the data, not all the quirks and fluctuations of the training sample; this way models will generalize better. Instead of an exact, "ideal", a "good enough" solution is favored, even when several runs on the same data, due to random effects, generate several similar, but not identical models. In ML courses often Ockham's razor, named for the fourteenth century Franciscan friar, is cited as a helpful path to generalizibility: "More things should not be used than are necessary." Based on our knowledge about physics, we can be less restrictive. As Albert Einstein famously said: "Everything should be made as simple as possible, but not simpler." Good ML models find a balance between the two. Once a model is trained, it can be applied on new data, the so called inference. Usually this step is much less computationally intensive, providing sizable speed-ups in processing data.

Early ML applications in HEP often used decision trees: a tree like model for decisions, starting at the root, climbing up the branches and reaching the leaves, where each leaf represents a decision. For classification problems, each leaf represents our decision assigning a data item to a class (binary or multiclass problems). In HEP, the most widely used are boosted decision trees (BDT), which convert "weak" to "strong" learners.

Artificial neural networks (ANN or just NN) try to imitate in a simplified way biological brains. The neurons and synapses are replaced with connected layers of nodes (units, or sometimes even simply neurons) and edges. A node takes inputs from its connections as real numbers (a weighted sum of the connected outputs from the previous layer), and performs a non-linear transformation to form its output. Typical activation functions for this are: sigmoid (logistic) and tanh where the output is limited below $|1|$ for any input values, and the rectified linear unit $R e L U$ $(\max (0, x)$ or the positive part of the argument). NN have an input, an output, and one or multiple ("deep learning"- DL) hidden layers. Deep NN are denoted as DNN.

The learning can be supervised based on pairs of inputs with known outputs for training, or unsupervised, for example density estimation, clustering or compression. A cost or loss function measuring the "distance" between the current and the 
desired outcomes is minimized to train the model. Classical optimization aims to minimize the cost function on the available (training) data, while in ML the goal is to generalize, or minimize the cost best, on the unseen (test) data. At each step the weights for all the edges can be adjusted by backpropagation based on the differentiation chain rule to reduce the cost function by small amounts. This is the stochastic gradient descent (SGD). The associated learning rate is similar to the $\epsilon$ introduced by Cauchy ${ }^{7}$ to formalize calculus in the nineteenth century.

Many familiar terms have their equivalents in ML jargon: variables are called features, iterations become epochs, labels often are called targets. To speed up convergence, minimizations are carried over data batches of limited size, and the weights adjusted, instead of traditional global solutions in one go, which are much slower. Multilayer architectures can be trained by backpropagation and SGD. The fears from local minima, unwanted e.g. in HEP fit applications, have largely dissipated. For complex phase spaces there are many saddle points which give very similar values of the cost function, i.e similar models. ${ }^{2]}$ Instead of SGD, a popular optimizer is Adam,$\frac{8}{}$ which adjusts the learning rates per parameters and based on recent history.

While the values of edge weights are learned during training, the so called hyperparameters, like learning rate, model architecture (e.g. number of hidden layers and nodes per layer), activation functions, or batch size, are set before one run of the learning cycle begins. Depending on the data patterns to be learned or abstracted, different values of the hyperparameters will be needed for the same ML tool. The hyperparameter tuning necessitates several, often many learning runs. Here is where human intervention and data scientist skills are key.

To keep this ML overview concise, more details about specific ML techniques will be provided throughout the text.

\section{Machine Learning in Experimental HEP}

\subsection{Classification and Event Selection}

The difficulty in extracting small signals from the towering LHC backgrounds has helped to introduce ML techniques for classification purposes. Classification algorithms are a type of supervised learning where the outputs are restricted to a limited set of values, or classes like signals or backgrounds. The Higgs analyses are a prime example.

The discovery of the Higgs boson in 2012 by the $\mathrm{CMS}^{9}$ and ATLAS ${ }^{10}$ collaborations saw the first use of boosted decision trees in such a high stakes search for the separation of small signals (invariant mass peaks) over large smoothly falling backgrounds. Since then the Higgs decays and couplings to the heavy W and Z gauge bosons, as well as the heavy third generation quarks (bottom and top) and tau leptons, have been observed by both ATLAS and CMS, and are consistent with the predictions of the SM at the current level of precision. With the Higgs boson firmly established, attention has turned to measuring its properties. 
The next frontier is observing Higgs decays and measuring its couplings to fermions outside the third generation. The search for Higgs decays to a pair of muons with opposite charge $\left(\mu^{+} \mu^{-}\right)$offers the best chance to establish and measure the Higgs couplings to the second generation. This is a very challenging undertaking: the SM branching fraction is expected to be $\sim 0.02 \%$. The small signal has to be extracted over a huge irreducible background producing opposite sign muon pairs: Drell-Yan, top quark or $\mathrm{W}$ boson pairs production. The Higgs signal has a narrow dimuon invariant mass peak near $125 \mathrm{GeV}$, only a few $\mathrm{GeV}$ wide, determined by the experimental muon momentum resolution. In contrast, the background events exhibit a smoothly falling mass spectrum in the search region from 110 to $160 \mathrm{GeV}$.

The CMS collaboration developed a method to enhance the signal extraction by using a BDT classifier, as implemented in the TMVA class $\frac{11}{11}$ of the ROOT analysis package $\frac{12}{12}$ augmented with automated categorization for optimal event classification. The results of the 2016 analysis, using $35.9 \mathrm{fb}^{-1}$ of collision data, were published in ${ }^{13}$ Details of how the analysis was optimized for maximum signal sensitivity, utilizing multivariate and machine learning techniques, are provided in! ${ }^{14}$ Events are divided into categories based on the transverse momentum $\left(p_{T}\right)$ of the dimuon pair (which is higher for the main gluon-gluon fusion signal $(\mathrm{ggF})$ relative to the main Drell-Yan background), or the presence of a high-invariant-mass dijet pair, characteristic of vector boson fusion (VBF) signal events. Categories are subdivided further based on the muon pseudorapidity $(\eta)$, as central muons have better $p_{T}$ resolution, resulting in a sharper signal mass peak.

The training is based on one million simulated events for the various channels, fully reconstructed in the CMS detector. Fourteen kinematic variables characterizing the dimuon system are used, their distributions are very similar between the signal and background events, making the separation that much harder. The signal sample is split into three independent sets: one for training, a second for testing, and a third completely independent - to avoid any bias - for the final measurement. The background samples are typically split in $75 \%$ for training and $25 \%$ for testing. A binary signal-background separation is computed, yielding a BDT score between -1 and 1 , where events close to 1 are more signal-like, and events close to -1 are more background-like.

As a last step the auto-categorizer procedure determines 15 event categories based on $|\eta|$ and BDT scores. Performing separate signal-plus-background fits to the data in all of these categories and combining the results significantly increases the search sensitivity relative to a measurement of all candidate dimuon events together. The net result of applying machine learning techniques is a $23 \%$ increase in sensitivity equivalent to $50 \%$ more data.

The ATLAS collaboration has presented an updated result $\frac{15}{15}$ using all the Run2 data: $139 \mathrm{fb}^{-1}$. The observed upper limit on the cross section times the branching ratio of the Higgs decay to a muon pair is 1.7 times the SM prediction, so the LHC experiments are closing in on the observation of this channel, but will need more data. 
This analysis follows a somewhat similar approach, using 14 kinematic variables and 12 categories to optimize the separation of the signal from the backgrounds. Data events from the sidebands and simulated signal events enter the BDT training procedure. The XGBoost (eXtreme Gradient Boosting) ${ }^{16}$ package is used. First a BDT is trained in the category of events with two or more jets to disentangle the VBF signal from the background. Three VBF categories with different purities are defined based on this BDT score. Then the rest of the events is divided with three BDTs providing ggF scores, and split according to jet multiplicities with zero, one or two jets, giving nine additional categories. To maximize the sensitivity to the Higgs to muons decays the boundaries are adjusted by BDT scores, and in each of the twelve BDT categories a fit to the invariant mass spectrum from 110-160 GeV is performed to extract the signal.

This analysis is able to achieve about $50 \%$ higher expected sensitivity compared to the previous ATLAS result, with roughly equal parts due to the increase in integrated luminosity or from refinements in the analysis techniques, where machine learning plays a key role.

A substantially more difficult task is the search for Higgs decays to a pair of charm quarks from the second generation. The CMS collaboration has performed a direct search for this Higgs decay where the Higgs is produced in association with a $\mathrm{W}$ or $\mathrm{Z}$ boson, based on an integrated luminosity of $35.9 \mathrm{fb}^{1}$ collected at the CERN LHC in $2016 ! 17$

Two types of jet topologies are analyzed: "resolved-jet", where both charm quark jets from the Higgs decay are observed, and the "merged-jet" topology, where the two jets from the charm quark can only be reconstructed as a single jet. In both topologies, novel tools based on advanced machine learning techniques are deployed.

For the "resolved-jet" topology BDT with gradient boost are trained to enhance the signal-background separation. Four categories having 0,1 or 2 leptons from the associated $\mathrm{W}$ or $\mathrm{Z}$ decays (the 2 lepton case subdivided depending on the $\mathrm{p}_{T}$ of the vector boson) and 25 input variables are used for training. For the "merged-jet" topology a novel algorithm based on advanced ML methods is deployed to identify jet substructures in order to tag the highly boosted W, Z, and Higgs decays, giving sizable gains.

The use of an adversarial network ${ }^{18}$ helps to largely decorrelate the algorithm from the mass of a jet while preserving most of its discriminating power. For example, for large jets with $\mathrm{p}_{T}>200 \mathrm{GeV}$, misidentification rates for charm quark pairs of $1 \%, 2.5 \%$ and $5 \%$ are achieved for efficiencies of $23 \%, 35 \%$ and $46 \%$. The corresponding b jet misidentification rates are $9 \%, 17 \%$ and $27 \%$.

The results of the two topologies help to provide an upper limit on the branching ratio of Higgs decays to charm quarks. There is still a long way to reach the sensitivity needed to observe this Higgs decay with SM strength.

While in the early Higgs papers BDTs were the prefered ML approach, newer analyses deploy deep learning and NN. The CMS collaboration has measured ${ }^{19}$ the inclusive cross section for the production and subsequent decay of Higgs bosons to 
tau lepton pairs with $77.4 \mathrm{fb}^{1}$ of data collected in 2016 and 2017.

A multi-classification approach is applied for each final state and year of datataking, eight independent tasks in total. For each of them a fully connected feedforward NN is trained. The architecture consists of two hidden layers with 200 nodes each, and five or eight nodes in the output layer, each representing an event class prediction, depending on the final state. The total cross section as well as cross sections for individual production modes and kinematic regimes are obtained. This is made possible by the power of classification using deep learning.

Another recent example is the measurement of associated production of top quark-antiquark pairs and Higgs bosons (ttH), with Higgs decaying to b quarks, by the CMS collaboration. ${ }^{20}$ The analysis is based on $41.5 \mathrm{fb}^{1}$ collected in 2017 , and combined with the 2016 analysis reaches an observed (expected) significance of 3.9 (3.5) standard deviations above the background-only hypothesis, providing the first evidence for $\mathrm{ttH}$ production with subsequent $\mathrm{H} \rightarrow \mathrm{bb}$ decays. Multiple classifiers are deployed, like BDTs for the dilepton channel, or feedforward NN with three hidden layers of 100 nodes each, as implemented in Keras, $\stackrel{21}{2}$ for the single lepton channel.

The application of ML techniques for Higgs analyses at the LHC is not a oneway street. Data from the simulations of the Higgs decays to tau-lepton pairs were released by ATLAS to the ML community and formed the basis for the HiggsML challenge ${ }^{[22}$ It ran from May to September 2014 on the Kaggle platform, ${ }^{23]}$ was extremely popular, attracted 785 teams with 1942 participants and generated 35772 submissions and more than a thousand forum posts. Probably more surprising then than now, first prize was won by Gabor Melis, a software developer and consultant from Hungary, using artificial NN. A special HEP meets ML award was provided to data science graduate students Tianqi Chen and Tong He for offering the boosted decision trees tool XGBoost, used by many participants. By now CERN provides an open data porta ${ }^{\sqrt{24}}$ to the LHC experiments to encourage fruitful collaboration between high energy physicists and data scientists.

Great progress in computer vision has come from convolutional neural networks $(C N N)$, inspired by the animal visual cortex, where individual neurons process information only from parts of the visual field. This "divide-and-conquer" strategy simplifies the $N N$ architecture and helps features like translational and rotational invariance, very desirable for image recognition. Typically the first layers of a CNN are for convolution and pooling. In a convolution the shape of an input function is modified by another function by taking an integral of the product of the two. The convolutional filtering helps e.g. in edge detection. Hand-engineering the filters is replaced by learning them from the images. Pooling layers combine the inputs from several neurons (the simplest being a $2 \times 2$ cluster) into one output neuron, thus reducing the dimensionality. This is usually followed by fully connected layers like in standard DNN for the final classification step.

Traditionally a HEP analysis proceeds to reconstructing higher level objects like tracks and energy deposits in electromagnetic and hadron calorimeters from the 
raw detector data, and finally arriving at particle level objects. A promising new approach is to apply deep learning algorithms directly to low-level detector data. This is explored in what is called end-to-end event classification $\frac{25}{25}$ The study is based on 2012 CMS Simulated Open Data for the decays of the Higgs boson to a pair of photons. The gluon-gluon fusion Higgs production is the signal, while irreducible quark fusion to photon pairs and a photon plus jet faking a second photon events form the backgrounds in this simplified study. The events are simulated taking into account the interactions in the detector materials and the detailed CMS geometry.

The low level detector data is converted into images of size 170x360 in pseudorapidity $\eta$ and azimuthal angle $\varphi$ for the CMS barrel, and two images of size 100x100 for the two CMS endcaps extending to $|\eta|<2.3$. Each channel contains three layers corresponding to electromagnetic and hadron energy and track transverse momentum. This is the electromagnetic-centric segmentation. Alternatively, a hadron-centric segmentation with size $280 \times 360$ in $\eta-\varphi$ is used. Inspired by the recent progress in computer vision, a CNN of the Residual Net-type (ResNet-15) is used. The initial results show promise, with signal efficiency and background rejection on par with more traditional approaches.

\subsection{Reconstruction}

Regression algorithms are another type of supervised learning, providing continuous outputs which can have any numerical value within a range. They can be deployed for reconstruction purposes in HEP e.g. when we want to make precise determinations of continuous quantities like hit positions, track momenta or jet energies.

At the intensity frontier advanced detectors collect record amounts of luminosity at what would be considered "medium" energies by today's standards. One example is the Beijing Electron Positron Collider (BEPCII) running at center of mass energies 2.0-4.6 GeV. The BESIII experiment has collected record size data samples in this $\tau$-charm region. Advanced ML techniques have been applied for many tasks 27 One of them is cluster reconstruction for the cylindrical triple-GEM inner tracker, part of the 2019 upgrade to the aging inner drift chamber. The goal is to measure the drift cathode layer position of ionizing particles from the readouts of the anode strips, which is the first reconstruction step. Two methods are available: weighted by electric charge average position of the anode strips (Q method), or time measurement using the drift gap as kind of micro time projection chamber ( $\mathrm{T}$ method). The two methods can be combined to improve the position resolution, but this combination is made difficult by the correlations to the incident angle. Here ML techniques come to the rescue: a XGBoost regressor is developed to reconstruct the initial particle positions from the $\mathrm{Q}$ and $\mathrm{T}$ readouts. Substantial improvements over the charge centroid method are reported.

ML techniques are entering in full force the "sister" field of particle astrophysics. One development in the field of very high energy gamma-ray astronomy is the Cherenkov Telescope Array (CTA) which will ultimately consists of 19 telescopes 
in the Northern and 99 telescopes in the Southern hemisphere to cover the full sky. A colossal amount of data in the multi-petabyte range per year is expected. The telescope arrays are operated as a single instrument to observe extensive air showers originating from gammas or charged particles, and aim to separate them and measure basic characteristics as energy, direction and impact point of the original particle. An exploratory regression study $2 \underline{28}$ in this direction uses CNN with the hope to extract more information directly from the raw data and outperform traditional approaches based on human-selected features.

The main difficulty is that conventional CNNs are developed to process rectangular images with regular pixel grids. The telescope outputs here have hexagonal pixels forming hexagonal images. One, not very satisfying approach is resampling, converting the image to a standard one, potentially losing some information about the neighbors. This analysis takes the more difficult route of reimplementing the convolutional and pooling operations of CNNs by building matrices of neighbor indices, rearranging the data accordingly and then applying the general methods for convolution, or for pooling with different functions depending on the task (softmax, average, $\max$ ).

The next difficulty is to combine images from several telescopes to obtain stereoscopic information. Traditional DL methods only deal with single images, sequentially in time. This is solved by adding a convolution block for each telescope in the array, and feeding them all to the dense fully connected part of the network. The exploratory study with four telescopes shows promise in the measurements of energy, direction and impact point for incoming particles; additional work is needed to outperform traditional methods and solve technical details before applying the developed algorithm on real data.

Tracking detectors form the core of most collider experiments, and successful track reconstruction is mission critical for achieving their goals. Reconstructing tracks is a combinatorial problem, i.e. finding the measurements (hits) belonging to individual particles entering the detectors from an often huge set of possible combinations. With the transition to the High-Luminosity LHC (HL-LHC) the complexity of this task will increase substantially. Traditional approaches like track following (inside-out or outside-in) and Kalman filters do not scale favorably to very high hit densities, and are typically custom implemented for each experiment with large amount of human efforts.

The TrackML ${ }^{29}$ project has the ambition to stimulate new approaches and the development of new algorithms by exposing data from a virtual, but realistic HLLHC tracking detector to data science and computer experts outside of the HEP community. Production of top-antitop quark pairs is selected for the signal events, which are then merged with 200 soft interactions (pile-up events). Fast simulation is used to generate hits in the tracker from charged tracks. The magnetic field is inhomogeneous, energy loss, hadronic interactions and multiple scattering are parameterized. The silicon tracker consists of three parts: innermost pixel detector, followed by two layers of short and long silicon strips providing hermetic coverage 
up to $|\eta|<3$. For each collision, about ten thousand charged particles, originating approximately from the center of the detector, produce about ten precise hits per track in three dimensions.

The challenge, running on the Kaggle platform ${ }^{30}$ and on Codalab in 2018-2019, is split in two phases: accuracy and throughput. The first phase is scored by a specially developed metric, which puts high priority on efficiency of finding real hits belonging to a particle and low fake rates. At least $50 \%$ of the hits have to originate from the same simulated truth particle, with hits on the innermost layers, key for good vertex resolution, and on the outermost layers, key for long lever arms and thus for good momentum resolution, getting highest weights in the overall score. A random solution will get a score of zero and a perfect reconstruction of all events in the test dataset, consisting of 125 simulated events, will get a score of one.

The challenge attracted more than 650 participants. In the accuracy phase the participants provide their reconstruction of the test dataset to Kaggle where it is scored. In the throughput phase the participants provide their algorithms and software, and it is run in a consistent environment (Docker containers on two i686 processor cores and 4GB of memory) to measure both accuracy and runtime, which will be very important to handle the enormous datasets expected from the HL-LHC.

Winners 31 of the accuracy phase are teams (with scores): top-quarks(0.92219), outrunner(0.90400) and Sergey Gorbunov(0.89416). While training can consume lots of computer resources, where machine learning really shines is the speed of reconstruction once the algorithms are trained. Winners of the throughput phase are teams sgorbuno (Sergei Gorbunov), fastrack (Dmitry Emelyanov) and cloudkitchen (Marcel Kunze), who were able to combine high accuracy scores with speeds well below ten seconds per event, and even below one second for the first two.

The TrackML challenge shows that ML techniques like representation learning, combinatorial optimization, clustering and even time series prediction can be applied to tracking. The best solutions offer a synergy between model-based and data-based approaches, combining the best of both worlds: physical track models and machine learning, with sensible trade-offs between complexity and performance.

\subsection{Particle Identification}

Particle and jet identification are examples where machine based classification methods are rapidly replacing the traditional HEP approaches.

The LHCb experiment at the LHC specializes in the physics of beauty quarks. Identifying the types of long lived charged particles in the tracker, ring-imaging Cherenkov detectors, electromagnetic and hadron calorimeters and the muon chambers is key. Global particle identification (PID) based on machine learning techniques is developed ${ }^{[32}$ The charged particle classes are: electron, muon, pion, kaon, proton and ghost track (fakes created by the tracking algorithm).

The baseline PID approach, ProbNN, is based on six binary (one-vs-rest) onelayer shallow artificial NN, implemented in the TMVA library. Each network sepa- 
rates one particle type from the rest. The DeepNN with three hidden layers of 300, 300 and 400 neurons is based on Keras, and works in multiclassification mode to separate the six particle types in one go. CatBoost consists of six "gradient boosting over oblivious decision trees classifiers", working in one-vs-rest mode. Sixty observables from the LHCb detectors are available for PID; DeepNN and CatBoost use all of them, while ProbNN uses different subsets for each PID hypotheses, based on physics reasons. The classifiers are trained on one million simulated events for each charged particle type.

The performance is verified on real data using kinematically identified decays to known particles like $J / \psi \rightarrow e^{+} e^{-}\left(\mu^{+} \mu^{-}\right), \Lambda \rightarrow p \pi^{-}, D^{0} \rightarrow K^{-} \pi^{+}$. The separation quality of the different classifiers is compared for six signal-background pairs: evs- $\pi$, e-vs-K, $\mu$-vs- $\pi$, K-vs- $\pi$, p-vs- $\pi$ and p-vs-K. Different classifiers score best for different pairs, with CatBoost and DeepNN, by using all observables, outperforming ProbNN on most counts. The proton-kaon separation is the most difficult, as both leave similar traces in all detector systems. Here using all the available information provides a clear advantage.

The Belle II experiment is operating at $\Upsilon(4 S)$ center-of-mass energy of 10.58 $\mathrm{GeV}$ at the SuperKEKB energy-asymmetric electron-positron B factory with record design luminosity of $8 \cdot 10^{35} \mathrm{~cm}^{-2} \mathrm{~s}^{-1}$, a factor of forty increase. This will expand the intensity frontier, with the size of the Belle II dataset expected to be fifty times bigger than the one collected by Belle. For the study of $\mathrm{CP}$ violation and flavor mixing in neutral B meson decays, the copious decays $\Upsilon(4 S) \rightarrow B^{0} \bar{B}^{0}$ are used. One of the $B$ mesons is fully reconstructed (signal side, including all products of this decay), and the flavor of the second (containing a b quark or antiquark) has to be determined (tag side, the rest of the particles). This is called flavor tagging.

To ensure the success of the physics program, improved flavor taggers using machine learning are developed ${ }^{33}$ to cope with the ultra high luminosity and increased beam backgrounds. A category-based tagger uses fast BDTs. A $B^{0}\left(\bar{B}^{0}\right)$ meson contains a positively charged $\bar{b}$ (negatively charged $b$ ) quark, which can decay e.g. to a positive (negative) lepton. Using multivariate analysis, thirteen specific categories are identified, where the flavor signatures of the measured decay particles are correlated with the $B$ meson flavor. Each category contains one or two particles: e, $\mu$, lepton (e or $\mu$ ), $\mathrm{K}, \pi, \Lambda \rightarrow p \pi^{-}$, called targets. PID variables from the various subdetectors and kinematic variables (simple like momenta and impact parameters, and global like the recoil mass) are used to identify the targets among all the target side particles. In a first step individual tag-side tracks are found, using 108 unique inputs. Each particle candidate receives 13 weights in $[0,1]$ for the probability of being the target for a category. The candidate with the highest weight for a category is selected as the target. The second step combines the outputs from the thirteen categories, again using multivariate methods. This improves the performance, as the $B_{t a g}^{0}$ decay can produce more than one flavor-specific signature, so more than one category will contribute.

The performance of the category-based flavor tagger is evaluated on simulated 
Belle II, and simulated and real Belle events. For the simulated events $B_{\text {sig }}^{0}$ decays to $J / \psi K_{s}^{0} \rightarrow \mu^{+} \mu^{-} \pi^{+} \pi^{-}$, while $B_{\text {tag }}^{0}$ has all possible decays. The sizes of the testing and training samples are 1.3 and 2.6 (1 and 2) million events for Belle II (Belle). Interestingly, the training sample has to be generated without $\mathrm{CP}$ violation to avoid the algorithm "learning" $\mathrm{CP}$ asymmetries on the tag side. The effective tagger efficiency on simulated events is $\sim 37 \%$, a $10 \%$ relative improvement over the Belle result. Larger training data samples give no further improvement. As an alternative, a deep-learning flavor tagger, based on a multi-layer perceptron (MLP) with eight hidden layers and 140 input variables is under development. It tries to learn the correlations between the tag-side tracks and the $B_{\text {tag }}^{0}$ flavor using the full information without any preselection of decay products. The first results are encouraging: while there is no improvement for Belle, the Belle II results indicate progress. The complexity of the MLP tagger requires huge training samples: the best results so far use 55 million events for training, and the tendency is to still improve with larger datasets. This computation takes about 48 hours with acceleration on a graphical GTX970 GPU, while the same training consumes about five hours on a single CPU for the category-based flavor tagger.

In many HEP measurements, identification of jet flavors is a key component. Traditionally this is done exploiting the characteristic features of heavy flavor charm or beauty hadrons, decaying at some distance from the primary interaction point. This produces displaced tracks and secondary vertices (SV), and often leptons from the sizable leptonic and semi-leptonic branching ratios. Additional difficulties arise from the embedding of the decay products within jets resulting from the parton shower. At collider energies these jets can often be highly boosted and collimated.

In the CMS collaboration the jet flavor classifier DeepCSV $\sqrt{34}$ (Combined Secondary Vertex) was developed. It uses a dense NN of five layers with 100 nodes each with ReLU activation, and an output layer with softmax activation to separate four classes: b, bb (two merging B hadrons in the jet), c and light (both quarks and gluons). The model is implemented in Keras with a TensorFlow ${ }^{355}$ backend. Sixtyeight input features enter the NN: 8 for each of the six most displaced tracks, 8 for the most displaced SV, and 12 global variables. Missing features are represented as zeros. Pile-up tracks, fakes and nuclear interaction vertices are rejected in advance. Notably, cMVAv2, the best previous tagger using additional lepton information, was outperformed by DeepCSV.

The success of deep learning in the jet arena sparked interest for more complex models in CMS,$\frac{36}{36}$ based on CNN. These networks have been used e.g. for classification of highly boosted jets, where the internal jet energy distribution is a major focus. The DeepJet algorithm for flavor identification applies CNN not on images, but on single particles. No preselection is needed. The input variables are 16 for up to 25 displacement sorted tracks, 8 for up to 25 neutral candidates, 12 for up to $4 \mathrm{SV}$, and 15 global for a total of up to 663 inputs. Passing through a set of convolutional layers, these produces 8,4 , and 8 features for each input track, neutral 
candidate or SV. The network automatically "engineers" and selects the relevant features. This way the large number of input variables can be handled efficiently by a "divide-and-conquer" strategy. The network architecture is shown in Fig. 1 .

\begin{tabular}{|c|c|c|c|c|}
\hline Charged (16 features) $\times 25$ & $1 \times 1$ conv. $64 / 32 / 32 / 8$ & RNN 150 & \multirow{4}{*}{$\begin{array}{c}\text { Dense } \\
200 \text { nodes } \times 1 \text {, } \\
100 \text { nodes } \times 5\end{array}$} & \\
\hline Neutral (8 features) x25 & $1 \times 1$ conv. $32 / 16 / 4$ & RNN 50 & & $\begin{array}{l}\text { bb } \\
\text { lepb }\end{array}$ \\
\hline Secondary Vtx (12 features) x4 & $1 \times 1$ conv. $64 / 32 / 32 / 8$ & RNN 50 & & c \\
\hline Global variables (15 features) & & & & g \\
\hline
\end{tabular}

Fig. 1. DeepJet network architecture. Image from reference ${ }^{36}$ under a CC BY 4.0 license (https://creativecommons.org/licenses/by/4.0/).

In recurrent neural networks (RNN) the connections between the nodes form a directed graph along a temporal sequence. The graphs can be cyclic or acyclic. Sequences of inputs can be processed by the same units (with same weights), giving the RNN a "memory". Besides helping with speech recognition, this architecture can process inputs of variable sizes, e.g. a changing number of tracks and jets per event. Long short-term memory (LSTM) is a special case of RNN with feedback connections, giving it a gated memory (state) for retaining information over longer and better controlled time intervals.

Three independent RNN continue the processing, producing compact summaries of dimensionality 150, 50 and 50 for the candidate types track, neutral or SV. These outputs are combined with the global variables to enter a dense NN of 1 layer with 200 nodes and 5 layers of 100 nodes each. A final output layer separates six jet classes: one B hadron, two B hadrons, leptonic B hadron decay (three b type jets), charm, light quark (uds) and gluon. The last layer has a softmax activation, all the other ReLU activation. DeepJet shows sizable improvements compared to DeepCSV, for example in $t \bar{t}$ events at high jet $\mathrm{p}_{T}>90 \mathrm{GeV}$ for $\mathrm{b}$ jet efficiency of $90 \%$ the number of false positives is suppressed from 10 to $3 \%$. At the same time, the light quark versus gluon discrimination is on par with dedicated RNN binary classifiers, and slightly better than CNN using jet images.

\subsection{Physics Measurements}

From a ML point of view the measurement of a continuous quantity is a regression problem. The use of ML for optimizing high precision measurements at the LHC is explored in $\sqrt[37]{5}$ The measurement of the forward-backward asymmetry $A_{F B}$ of lepton pairs en route to the precise determination of the electroweak mixing angle using linear (LR) or deep NN regressors (DNNR) is developed as a test case. The question addressed is whether neural network regressors based on ML can perform on par 
(or even better) with the traditional HEP approach, based on our knowledge of how things have been done for decades? The beaten path of measuring $A_{F B}$ is to obtain the $\cos \theta$ distribution of the electron or negative muon in the Collins-Soper frame.$^{38}$ To this end the kinematic variables undergo a Lorentz transformation to this frame, and then forward and backward events are identified or a fit is performed to extract the asymmetry, and ultimately the electroweak mixing angle.

The analysis is performed in narrow regions at different invariant masses for the final-state lepton (dielectron or dimuon) pairs around 70, 91, 200 and $500 \mathrm{GeV}$, chosen to follow the change of the asymmetry $A_{F B}$ with mass from negative values below the $\mathrm{Z}$ peak to positive values above the peak. The event sample, simulated with the PYTHIA ${ }^{39141}$ generator, consists of 3991 events after acceptance cuts for a generic LHC detector in the region up to $|\eta|<2.4$ : $75 \%$ for training, $10 \%$ for validation and $15 \%$ for testing. The input variables for the ML regressors are: dilepton invariant mass $m$, transverse momentum $p_{T}$ and rapidity $y$ of the dilepton system, pseudorapidity $\eta_{1 o r 2}$ and transverse momentum $p_{T}^{1 \text { or } 2}$ for each decay lepton. As the goal is to extract the asymmetry from the decay kinematics and the angles "hidden" inside it, the invariant mass, which relates directly to the forward-backward asymmetry $A_{F B}$ in narrow mass regions, is not used. All input variables are transformed to span the range $[-1,1]$ in order to help the minimization procedure. The target variable is the observed forward-backward asymmetry $A_{F B}$.

Experimentation shows that the most sensitive input variables are the rapidities. To account for the symmetric nature of the LHC, where the observed $A_{F B}$ is stronger when the dilepton system has higher boost away from the center, the rapidities are converted to derived features (also called synthetic features in ML), such as the absolute value of the rapidity $|y|$ and of the pseudorapidities $\left|\eta_{1}\right|,\left|\eta_{2}\right|$. The training of the models is performed over two thousand epochs. The performance is quantified by the root mean squared error (RMSE) between predictions and targets for all events. A DNNR has better chances to learn the non-linearities in the dataset at the price of introducing higher complexity with more parameters to be determined at the learning stage. A NN architecture well tuned to the problem was selected: two hidden layers with ten nodes each, $R e L U$ activation and Adam optimizer.

For each mass region the "measured" $A_{F B}$ is extracted from the ensemble of all predictions from the events in this region. To minimize the impact of a few outliers (predictions far from the simulated "truth"), the median for each region is taken. The RMSE $\mathrm{E}_{\text {asym }}$ between the so determined asymmetries and the four true asymmetries provides a performance measure for each model training. The traditional high energy physics way of doing the analysis by counting forward and backward events gives RMSE $\mathrm{asym}_{\text {as }}=0.072$, the LR 0.099 and the best DNNR 0.082. After normalizing the input features the LR provides a useful starting point. The best performance with a DNNR is only $14 \%$ worse relative to the traditional way, which is an impressive result given the small size of the learning dataset for this 
prototype. It is reasonable to expect that by increasing considerably the size of the simulated samples and by tuning further the architecture and hyperparameters of DNNR for optimal performance, the ML results can improve and be at least on par with the traditional approach.

\subsection{Simulations}

Generative Adversarial Networks 42 (GAN) are a promising recent invention. A discriminator (often a CNN) is trained to recognize items from a training set. Then a generator (often a deconvolutional NN) generates samples with the goal of making them indistinguishable in statistical sense from the training set. The two NN play an "adversarial game": the generator tries to fool the discriminator by making it falsely classifying more and more synthetic items as true. During training, the generator tries to increase its success at fooling, while the discriminator tries to get better at rejecting the synthetic items. At the end of the game the true and synthetic distributions should be hard to tell apart.

GANs have been used for the production of 2D representations of energy depositions from particles interacting with a calorimeter - jet images, 43 and for fast electromagnetic shower simulation $\underline{44}$ directly producing component read-outs in a three-dimensional multi-layer sampling LAr calorimeter. Here attempts to apply similar techniques to tracking detectors are reviewed.

The ALICE experiment specializes in the study of heavy-ion collisions at the LHC. These collisions produce the most complex and highest density events. The prospect to use GANs for simulating clusters (space-time points) produced by particles in the Time Projection Chamber (TPC) of size 5x5x5 meters are explored in $\frac{45}{} \mathrm{~A}$ dataset of $3 \mathrm{D}$ trajectories for proton-proton collisions at center-of-mass energy $7 \mathrm{TeV}$, corresponding to the 2010 data taking, is generated. From 300 events corresponding to one million data samples, 54872 are retained: 40000 for training and the rest for testing. Each data sample contains the mass, charge and initial 3D momenta for a particle, together with all the clusters in the TPC. Two types of GANs are used: conditional Deep Convolutional GAN (condDCGAN), and conditional LSTM GAN (condLSTMGAN). The former employs multi-layer network with $2 \mathrm{D}$ convolutional/deconvolutional layers, the latter multi-layer recurrent network with LSTM units to process recursive data. The conditional approach adds the initial information about the simulated particles to the network. In addition the loss function, indicated by a plus sign in the model name, is modified to take both the initial parameters and the deviations of the clusters from the true trajectory into account.

The TPC resolution is between $0.8-1.2 \mathrm{~mm}$. The performance of the GANs is measured by the RMSE of the generated clusters from the trajectory approximated as an arc (part of a helix). For the traditional HEP full detector simulation RMSE is $1.2 \mathrm{~mm}$. For a condDCGAN+ model the speedup is 25 times with RMSE $=137 \mathrm{~mm}$, and for a condLSTMGAN+ it reaches 100 times for RMSE $=222 \mathrm{~mm}$. While the 
speedups are promising, clearly a lot of work is needed to improve this prototype before reaching a more optimal point on the speedup-accuracy curve.

\subsection{Anomaly Detection}

Quality monitoring is a key step in assuring that only good data where all the systems perform as expected enters the physics analysis chain, while bad data is detected and marked. At the same time the discarding of good data is not desirable. At the LHC this involves detector and online experts, and is time consuming. In the CMS experiment this is done by comparing many histograms filled with critical detector quantities to references collected at normal working points. Currently histograms are collected over runs, which can last from seconds to few hours. This makes certification more difficult, as transient problems over shorter periods may be difficult to track.

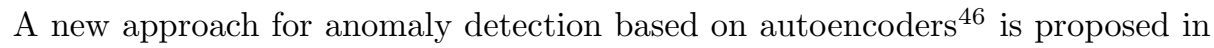
CMS. At the LHC, a luminosity section (LS) is defined by a fixed number of proton orbits in the accelerator, and lasts $\sim 23$ seconds. Each LS has a unique label within the run, incrementing from number one. The goal is to provide data certification at the LS granularity using machine learning. In CMS 401 control histograms from the various subsystems are monitored. Each of them is used to determine five quantiles plus mean and standard deviation, which results in 2807 input variables for ML. As anomalies typically are only $2 \%$ of the dataset, it is problematic to apply classical supervised ML classifications like feedforward NN. Also, the failures can be of many types or new ones, so it is difficult to collect large enough samples to train for sizable percentage of them. To handle this a semi-supervised approach is developed, where a model learns to detect only one class - the good one. Even unseen future detector failures can be caught this way!

This is implemented as an autoencoder (AE), which provides a map from inputs to their representations through an artificial NN. First an encoder produces a representation from the inputs. Then a decoder uses the representation (which typically has lower dimensionality) attempting to reconstruct the original input. An analogy is lossy compression. Early AE applications focused on training to improve compressed representations, but AE perform well to spot anomalies. When the inputs deviate from the "good" type, the representation suffers and the decoder output deviates from the original, signaling problems.

For this prototype 163684 samples from the 2016 data taking period are used. They are split in $60 \%$ for testing, $20 \%$ for validation, and $20 \%$ (used only once at the end) for testing. Experts removed "by hand" all anomalies from the training and validation samples. The network architecture has five hidden layers with 2000, 1000, 500, 1000 and 2000 neurons. Layers $1-3$ and 3-5 correspond to the encoding and decoding phase. The network architecture is shown in Fig. 2,

The anomalies are easily spotted by much larger MSE of their outputs from the inputs. As an added bonus the automatic detection also provides interpretability, 


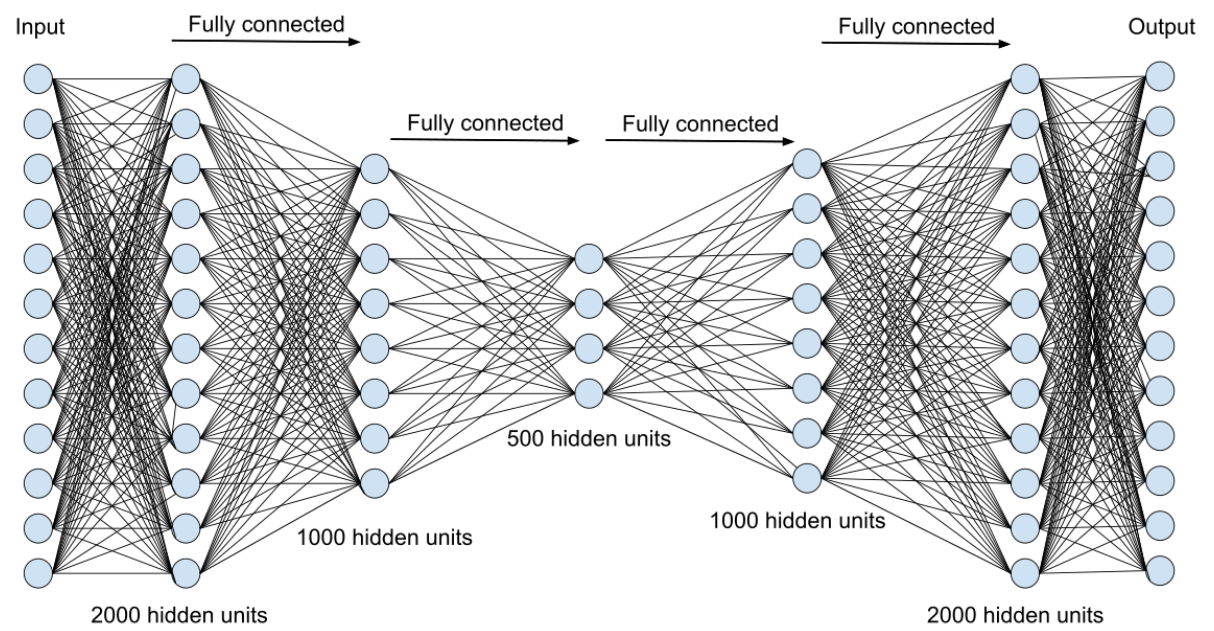

Fig. 2. Autoencoder network architecture. Image from reference $\frac{46}{4}$ under a CC BY 4.0 license (https://creativecommons.org/licenses/by/4.0/).

as it points to the subsystems with largest contributions to the MSE. Future work will focus on optimizing the model (hyperparameter tuning) and applying it for all Run2 data and for future LHC runs.

\subsection{Ultrafast Processing for $\mathrm{HL}-\mathrm{LHC}$}

Run3 of the LHC, scheduled to begin in 2021, will bring increased luminosities. The bunch crossing rate will be $40 \mathrm{MHz}$.

The LHCb experiment will upgrade the whole tracking system at the heart of their physics program. An upgraded Vertex Locator (VELO) will replace silicon strip detectors with higher resolution pixels. The pixel size is $55 \mu \mathrm{m}$, starting only $5 \mathrm{~mm}$ from the interaction point. This is expected to produce an excellent impact parameter resolution of $20 \mu \mathrm{m}$. LHCb is embarking on a radically new data-taking approach: a trigger-less readout, based on a software event filter and capable to handle the $40 \mathrm{MHz}$ rate. The background suppression is dominated by the particle tracks and displaced vertices reconstructed in the VELO.

To address this challenge, ML approaches are developed ${ }^{47}$ The raw data for each event stores information about all pixels that fired. Track reconstruction finds pixels belonging to unique particle trajectories. The baseline, traditional in HEP, algorithm starts from pixels, forms clusters of neighbors, then track seeds, cluster doublets, track segments, propagates them through the detector, adds more space points, etc. The forming of doublets suffers from combinatorial explosion in the high density LHC environments. Even starting from outside-in (from lower density) and looking for tracks pointing to the interaction region, the compute times are 
challenging. Overall, the baseline algorithm performs well in quality and speed. But finding the optimal values for the many tunable parameters, like search windows, is far from trivial, and porting to GPU accelerator architectures is difficult.

A ML approach is explored to reduce the track seed combinatorics: TrackNN, a fully connected NN (FCNN) with five layers. The non-linear activation functions are $R e L U$ and sigmoid for the last layer. Acting as a classifier, for each space point on a detector layer the FCNN finds among $\mathrm{N}$ space point the one with the highest probability to form a track segment on an adjacent detector layer, working outside-in. TrackNN reconstructs starting from the outer layers, only retaining track extensions when the probabilities pass quality thresholds, working towards the interaction region.

Simulated minimum bias events are used for training and testing. The performance is measured by the reconstruction efficiency and the rate of "ghosts" (spurious tracks) and "clones" (tracks reconstructed more than once). For the outermost 20 planes out of 50 total, TrackNN is almost on par with the baseline (efficiency 98.0 versus $98.9 \%$ ), with similar low ghost and clone rates. Moving in to using 30 and all 50 planes, the efficiency degrades to 93.2 and $71.1 \%$, and the ghost rate more than doubles. It gets harder and harder to find the real track segments closer to the origin. So far this purely ML-based prototype uses no a-priory knowledge about the physics of charged particle tracks or about the detector geometry. Incorporating this and with sustained efforts it may become possible to improve the reconstruction quality and to benefit from substantial speed-ups available on massively parallel architectures and on GPUs.

\subsection{Effectively Increasing the Luminosity}

An interesting aspect of the development of more powerful ML methods, outperforming the "traditional" ways of doing analysis, is the effective increase of the amount of data available to the experiments. For example, in the CMS search for Higgs decays to dimuons, $\frac{14}{14}$ the net result is a $23 \%$ increase in sensitivity equivalent to $50 \%$ more data. Similar result is reported by ATLAS, ${ }^{15}$ where about half of the $50 \%$ increase in sensitivity comes from refined analysis techniques based on ML, and the other half from increased luminosity. Perhaps on the optimistic side, even bigger gains for specific searches are expected ${ }^{\sqrt{48}}$ in phenomenological studies, described in the next section.

\section{Machine Learning in Theoretical/Phenomenological High Energy Physics}

Building upon the sustained successes of the SM in describing the measured phenomena in HEP, new hybrid approaches are developed pairing the strength of cuttingedge machine learning techniques with our knowledge of the underlying physics processes. 


\subsection{Constraining Effective Field Theories}

New data analysis techniques, aimed at improving the precision of the LHC legacy constraints, are developed in ${ }^{48}$ Traditionally in HEP, searches for signatures of new phenomena or limits on their parameters are produced by selecting the kinematic variables considered to be most relevant. This can effectively explore parts of the phase space, but leave other parts weakly explored or constrained. By using the fully differential cross sections at the parton level, approaches like the matrix element method or optimal observables can improve the sensitivity in the complex cases of multiple parameters. The weak side of these methods is how to handle the next steps to reach the experimental data: parton showers and detector response. Both of these steps are often simulated by complicated Monte Carlo programs with notoriously slow convergence of the underlying integrals. While simulations can be very accurate, they produce no roadmap how to extract the physics from data, especially for high dimensional problems with many observables and parameters. Building upon our knowledge of the underlying particle physics processes and the ability of ML techniques to recognize patterns in the simulated data, it can be effectively summarized for the next steps in the data analysis. In this way NN can be trained to extract additional information and estimate more precisely the likelihood of the theory parameters from the MC simulations.

The likelihood $\mathbf{p}(x \mid \theta)$ of theory parameters $\theta$ for data $x$ can be factorized in HEP as follows:

$\mathbf{p}(x \mid \theta)=\int d z_{\text {detector }} \int d z_{\text {shower }} \int d z \mathbf{p}\left(x \mid z_{\text {detector }}\right) \mathbf{p}\left(z_{\text {detector }} \mid z_{\text {shower }}\right) \mathbf{p}\left(z_{\text {shower }} \mid z\right) \mathbf{p}(z \mid \theta)$

where $\mathbf{p}(z \mid \theta)=\frac{d \sigma(\theta) / d z}{\sigma(\theta)}$ is the probability density of the parton-level momenta $z$ on the theory parameters $\theta$. The other terms in the integral correspond to the path from partons through parton showers, detector and reconstruction effects to the experimental data $x$ used in the analysis. The steps on this path have the Markov property: each one only depends on the previous one. A single event can contain millions of variables. Calculating these integrals, and then the likelihood function and the likelihood ratios, the preferred test statistic for limit setting at the LHC, is an intractable problem. On the other hand, at the parton level $\mathbf{p}(z \mid \theta)$ can be calculated from the theory matrix elements and the proton parton distribution functions for arbitrary $z$ or $\theta$ values. In this way more information can be extracted from the simulation than just generated samples of observables $x$, namely the joint likelihood ratio $r$ and the joint score $t\left(x, z \mid \theta_{0}\right)$ (which describes the relative gradient of the likelihood to $\theta$ ):

$$
r\left(x, z \mid \theta_{0}, \theta_{1}\right)=\frac{\mathbf{p}\left(z \mid \theta_{0}\right)}{\mathbf{p}\left(z \mid \theta_{1}\right)}
$$

The joint quantities $r$ and $t$ depend on the parton level momenta $z$, which for sure are not available in the measured data. Here ML helps by using suitable loss functions based on data available from the simulation to train a deep NN with stochastic 
gradient descent to approximate functionals that can produce the important likelihood ratio: $r\left(x \mid \theta_{0}, \theta_{1}\right)$ depending only on the data and theory parameters. For technical details we refer interested readers to $\underline{48}$ and references therein.

As a case study the weak-boson-fusion Higgs production with decays to four leptons is taken. The RASCAL technique uses the joint likelihood ratio and the joint score to train an estimator for the likelihood ratio. In essence this is a ML version of the matrix element method, replacing very computationally intensive numerical integrations with a regression training phase. Once the training is complete, it takes microseconds to compute the likelihood ratio per event and parameter point. As a bonus, the parton shower, detector and reconstruction effects are learned from full simulations instead of retorting to simplified, and sometimes crude, smearing functions. At the cost of a more complex data analysis architecture, the precision of the measurements is improved by tapping the full simulation information. For a typical operating point from the case study, aimed at putting limits on dimensionsix operators in effective field theories, a relative gain of $16 \%$ on the new physics scale is observed, corresponding to $90 \%$ more collected data.

\subsection{Model-Independent Searches for New Physics}

So far, searches for beyond the SM (BSM), new physics (NP), phenomena at the LHC have been negative, despite herculean efforts by the experiments. The majority of these searches are inspired and guided by particular BSM models, like supersymmetry or dark matter (DM). An alternative approach, which could provide a path to NP, potentially even lurking so far unseen in the already collected data, are model-independent searches. They could unravel unpredicted phenomena, for which no models are available.

One proof-of-concept ${ }^{49}$ strategy along these lines is developed based on unsupervised learning, where the data are not labeled. The goal is to compare two D (usually high) dimensional samples: the SM simulated events (background to BSM searches), and the real data, and to check if the two are drawn from the same probability density distribution. If the density distributions are $p_{S M}$ and $p_{\text {data }}$, the null hypothesis is $H_{0}: p_{S M}=p_{\text {data }}$, and the alternative is $H_{1}: p_{S M} \neq p_{\text {data }}$. In statistical terms, this is a two-sample test, and there are many methods to handle it. Here, a model-independent (no assumptions about the densities), non-parametric (compare the densities as a whole, not just e.g. means and standard deviations) and un-binned (use the full multi-dimensional information) two-sample test is proposed. As the densities $p_{S M}$ and $p_{d a t a}$ are unknown, they are replaced by the estimated densities $\hat{p}_{S M}$ and $\hat{p}_{\text {data }}$. A test statistic (TS), based on the Kullback-Leibler KL divergence measure,$\sqrt[50]{ }$ is built for the ratio of the two densities, with values close to zero if $H_{0}$ is true, and far from zero otherwise. The ratio is estimated using a nearest-neighbors approach. A fixed number of neighbors $\mathrm{K}$ is used, and the densities are estimated by the numbers of points within local spheres in $\mathrm{D}$ dimensional space around each point divided by the sphere volumes and normalized to the total 
number of points. Then the distribution of the test statistic $f\left(T S \mid H_{0}\right)$ is derived by a resampling method known as the permutation test, by randomly sampling without replacement from the two samples under the assumption that they originate from the same distribution, as expected under $H_{0}$. Accumulating enough permutations to estimate the TS distribution precisely enough, this allows to select the critical region for rejecting the null hypothesis at a given significance $\alpha$, e.g. 0.05, when the corresponding p-value is smaller than $\alpha$.

A proof-of-concept case study for dark matter searches with monojet signatures at the LHC is performed. The DM mass is $100 \mathrm{GeV}$, the mediator masses 1200-3000 $\mathrm{GeV}$, detector effects are accounted for by fast simulation, and the input features have $\mathrm{D}=8: p_{T}$ and $\eta$ for the two leading jets, number of jets, missing energy, hadronic energy $H_{T}$, and transverse angle between the leading jet and the missing energy. The comparison is done for $\mathrm{K}=5$ and 3000 permutations. As an added bonus, regions of discrepancy can be identified for detailed scrutiny in a model-independent way. The results show promise. Before applying them to real data, several refinements are needed: systematic uncertainties and limited MC statistics will weaken the power of the statistical tests, and the algorithm has to be optimized or made completely unsupervised by automatically choosing the optimal parameters like the value of $\mathrm{K}$.

A different approach ${ }^{\sqrt{51}}$ for NP searches based on supervised learning builds upon the same setup. This time, using the same notation as for the unsupervised approach introduced earlier:

$$
p_{\text {data }}(x \mid \mathbf{w})=p_{S M}(x) \cdot \exp f(x ; \mathbf{w})
$$

where $x$ represents the d-dimensional input variables, $\mathcal{F}=\{f(x ; \mathbf{w}), \forall \mathbf{w}\}$ is a set of real functions, and the NP would traditionally depend on a number of free parameters $\mathbf{w}$, introducing model dependence. Here $\mathcal{F}$ is replaced by a neural network, in effect replacing histograms with $\mathrm{NN}$, based upon their well known capability $\sqrt{52}$ for smooth approximations to wide classes of functions. The NP parameters are replaced by the NN parameters, which are obtained from training on the data and SM samples. The minimization of a suitable loss function (which also maximizes the likelihood) provides the best fit values $\hat{\mathbf{w}}$. Again a t-statistic and p-values are derived for rejecting the same null hypothesis, as well as the log-ratio of the data and SM probability density distributions.

The method is illustrated on simple numerical experiments for the resonant and non-resonant searches for NP in the 1D invariant mass distributions, and for a $2 \mathrm{D}$ case adding the $\cos \theta$ of the decay products.

A limitation of these methods is the precision of the SM predictions. Usually produced by MC full detector simulations, they are computationally costly. In addition, systematic uncertainties of the predictions reduce the sensitivity to new phenomena. Given the excellent performance of the LHC and the experiments, by the end of Run2 the data available in many corners of the phase space exceeds the MC statistics, and the situation could get even more critical in the future. Certainly approaches driven by data in relatively NP-free regions, e.g. sidebands of 
distributions, will also have an important role to play.

\subsection{Parton Distribution Functions}

The well known capability of NN for smooth approximations to wide classes of functions is used in Parton Distribution Function (PDF) fits to the available lower energy and LHC data by the NNPDF $53 \sqrt[54]{5}$ collaboration. The fit is based on a genetic algorithm with a larger number of mutants to explore a larger portion of the phase space, and nodal mutations well suited for the NN utilized as unbiased interpolators of the various flavors of PDFs. To avoid overfitting, the cross-validation runs over a validation set which is never used in the training, but remembers the best validation $\chi^{2}$. At the end, not the "best" fit on the training set, but a "look-back" to the best validation fit is retained as the final result. The NNPDF sets are easily accessible through the LHAPDF $55+58$ libraries.

A set of Monte Carlo "replicas" is used to estimate the uncertainties by computing the RMSE of predictions for physical observables over the ensemble. In practice this works well in most cases. Care is needed in corners of the phase space, like searches at high invariant masses, where cross sections for some members of the standard PDF set can become negative, or unphysical. For these cases, a special PDF set with reduced number of replicas, but ensuring positivity, is provided. The price to pay is enhanced PDF uncertainty compared to other PDF families, where the PDF parameterizations extrapolate to such phase space corners with smaller uncertainties. In any case, comparing several families before claiming a discovery is highly recommended.

\section{Challenges}

\subsection{Hyperparameter Tuning}

An area where the data scientist expertise is key is finding the optimal settings and the best ML architecture for the task at hand. Usually this requires many trials and errors before converging on a successful approach. Ways to optimize this process with an eye on multi core and multi GPU applications is explored in 59 Using an example for the CPU intensive process of training a GAN for a 3D calorimetry simulation, the authors first explore ways of parallelization on multiple nodes and/or multiple GPUs, and then turn to the task of hyperparameter optimization. Here the previously defined loss functions are not necessarily used as a figure of merit (FOM) to quantify the performance. Two approaches are studied: Bayesian Optimization with a Gaussian Process prior and an Evolutionary Algorithm. In the first one the FOM is modeled with Gaussian processes, and by sampling the often big hyperparameter space, successively better suggestions closer to the optimum are discovered. In the second approach the hyperparameters are optimized as the "survival" fitness of chromosomes. The initial results show speed-ups of around 8 times for 20 , and 20 times for 100 processes. The drop from linear speed-up will require additional work to reduce the sizable overhead. 


\subsection{Systematic Uncertainties}

The large amounts of data collected at colliders like the Large Electron-Positron collider (LEP) or the LHC, and at the intensity frontier, mean that the statistical errors on the collected data samples tend to get quite small, and often the systematic effects become important and even limiting. Experience shows that a large, often dominating amount of time in data analysis is spent on estimating and handling the systematic errors, after the express production of first, exploratory, results.

With the wide-spread use of ML techniques, handling of the systematic errors comes into play ${ }^{60}$ In this study simulated data from the HiggsML challenge is used to optimize the selection of Higgs decays in a typical supervised classification problem, where training is done on labeled examples of simulated data. If $\mu$ is the ratio of the number of measured signal events over the expected number, the FOM here is the relative error of $\mu$. It depends on the true positive (signal classified as signal) and false positive (background classified as signal) numbers of events. The FOM has a statistical and a systematic error. The latter comes from known nuisance factors making the test data different form the training data, e.g. accuracy of theoretical background predictions, detector efficiencies and calibrations affecting simulated (training) and real (test) data differently.

Three methods of systematic aware learning to minimize the FOM are explored: data augmentation, pivot adversarial network (very difficult to train), and knowledge integration. In this study the standard NN gives the best FOM. This may be due to the fact that reaching the FOM minimum requires to reject lots of data to obtain high signal purities, while the systematic aware methods become robust on the whole dataset. More work lies ahead to make them competitive.

\subsection{Sculpting}

A serious problem for the application of ML techniques in HEP is the sculpting of variables under study, like mass or $p_{T}$ distributions, which is an obstacle in a physics analysis. One example is the mass sculpting observed when applying the DeepDoubleB classifier ${ }^{36}$ To decorrelate the classifier output from the jet mass, two Kullback-Leibler divergence penalty terms are applied: between the original and the classifier output ("sculpted") distributions for the signal and for the background. In this case the the mass decorrelation has minimal impact on the classifier performance.

Another example ${ }^{32}$ is the sculpting of PID information as a function of kinematic variables like particle pseudorapidity, total and transverse momentum, and event multiplicity. To reduce the systematic uncertainty for physics measurements it is helpful to have a flat efficiency for PID. A specific BDT Flat 4d model, utilizing a modified loss function, is developed to guarantee the flatness of the efficiencies. Similar to CatBoost and Deep NN, the Flat 4d models uses the sixty observables from all $\mathrm{LHCb}$ systems. The flatness comes at the price of a somewhat decreased PID quality. 


\subsection{Speed}

The trigger requirements of the HL-LHC necessitate in some cases the deployment of field-programmable gate arrays (FPGA), integrated circuits which can be custom configured after manufacturing. They contain arrays of programmable logic blocks and memory elements, in order to make them flexible for implementing e.g. ultra-fast trigger algorithms, which would be traditionally implemented in standard computer software running on slower well known hardware configurations. The study ${ }^{61}$ is one example of exploring the challenges of ML with FPGA.

\section{Two-way Street: Machine Learning Meets Physics}

As demonstrated in the previous sections, ML and DL techniques can be beneficial for a wide scope of tasks in particle physics. It works in both directions: insights from physics can help to understand better how ML and DL operate, and potentially to design better network architectures. In addition, our knowledge of the underlying physics laws and the datasets collected or simulated by the experiments provide a more organized environment to study ML compared to the floods of data generated e.g. by the social networks.

\subsection{Insights from Statistical Physics and Information Theory}

$\operatorname{In}[\sqrt[62]{63}$ an intriguing approach to understanding ML based on information theory and statistical physics is explored. Similar to thermodynamics, complex many dimensional systems are described with only a few parameters like temperature and pressure. For a supervised network with an input, output and several hidden layers, just two numbers summarize the network state for each layer, relating it to the information from the input layer (encoding), and to the labels in the output layer (decoding). In other words, each layer compresses or extracts relevant features from the inputs and connects them better and better to the desired targets.

For feedforward DNN with sigmoid activation, interesting patterns depending on the increasing number of epochs are observed. The signal-to-noise ratio (SNR) exhibits 2 phases:

- High SNR: high mean of gradients and small standard deviation: linear drop (improvement) in accuracy, memorization of input features

- Low SNR: standard deviations for gradients bigger than means; diffusion, random walk and forgetting irrelevant dimensions.

Modern DNN can have millions of parameters and gradients. Typically the gradients are large in relatively few, important, dimensions, and flat in many, irrelevant dimensions. An apt metaphor is the Rio Grande Canyon cutting the plane near Taos in the state of New Mexico. For exact solutions the gradients are close to zero in the irrelevant dimensions. The SGD method, described earlier, has noise and variations when the batch sizes are small. As different layers hold information about different 
important dimensions, these studies propose that this actually helps. By adding noise and fluctuations to irrelevant dimensions, "diffusion" helps us to forget them, filters them out, thus aiding the convergence. SGD eliminates irrelevant features and compresses the information for relevant features. In this information theoretic view of layers, compression is at best weakly dependent on the non-linearity, and happens for all kinds of non-linear cases. This "compression theorem" depends strongly on the properties of diffusion. Through the separation in relevant and non-relevant features, the dimensionality of the problem is effectively determined not by the number of weights to be fitted, but by the number of relevant dimensions.

For smaller input datasets, the estimation of the covariance matrix is distorted by noise, thus including to some extent the non-relevant dimensions. Overfitting is determined by the local properties of the minimum and the corresponding weights. When there is "noise", the minimum is rotated somewhat from the relevant to the non-relevant dimensions (continuing the metaphor: like small tributary canyons to the main canyon). Diffusion for the relevant dimensions, supposedly "protected" by steep gradients (canyon walls), leads to decrease of information about the labels, and to overfitting.

To summarize, the somewhat "cavalier" attitude of data scientists towards minimization, developed through experimentation and intuition as mentioned in the introduction, appears to have quite solid foundations in statistical physics. If we "forget" about the non-relevant dimensions, there is not a single "best" solution, but a whole continuum of "close enough" solutions, which plateau in the nonrelevant dimensions. This ensures the often observed good generalizability of the SGD method.

\subsection{Insights from Theoretical Physics for CNN}

The concepts of covariance from theoretical physics, explored by Einstein while developing the special and general theory of relativity, are applied in $\frac{64}{6}$ to improve the CNN performance for image recognition. The coordinate independence was applied first for inertial frames through Lorentz transformations, and then generalized for local changes of coordinates which are space and time dependent. Not surprisingly, such features are very desirable for image recognition as sought for when developing $\mathrm{CNN}$ : we want the results of identifying images to be insensitive to translations, rotations, mirror reflections or even deformations of the same underlying patterns. The information coming out of the network can be equivalent for transformed images if the network architecture is equivariant under the corresponding group action. This ideas are inspired from renormalization group theory. In analogy to gauge theory, the transformation laws look similar to gauge transforms, but not quite, as they act on different space layers. Adding symmetries to DL enables the successful use of CNN not only for the traditional domain of two-dimensional images, but also on arbitrary manifolds, e.g. the three-dimensional sphere as viewed by the cameras of a flying drone. 


\subsection{Interaction Networks}

A novel architecture, interaction networks ${ }^{65}$ (IN), is developed by DeepMind Technologies UK, acquired by Google in 2014. The ambitious goal is to evolve methods for reasoning about physics, objects, and their relationships, in ways bringing AI closer to human intelligence. Capabilities like reasoning about object interactions in complex systems, abstraction of properties and dynamical predictions are developed. These capabilities are challenging, because the often large amounts of inputs can be combined in many possible, and not obvious, ways. The IN aims to do this by building models decomposing the complexity into objects and relations, using graphs, simulation and DL.

Objects are nodes in the network, and relationships are edges between nodes, which can be uni- or bi-directional. Implementations based on DNN with gradientbased optimization are possible. By separating the objects and their interactions, many algorithms can be implemented, without any need for ordering or preprocessing the inputs $\frac{65}{6}$ Case studies in the physics domain like n-body systems, and rigid or non-rigid dynamics, show that IN generalize well, even extending to novel, previously unseen systems with variable configurations and numbers of objects and relationships. In this sense a IN is a general tool showing "elements of reasoning".

IN are finding promising applications in jet physics. A jet identification algorithm ${ }^{66}$ is developed for the identification of all hadronic decays of high-momentum heavy particles. The goal is to distinguish between quark and gluon jets, producing one collimated cone of particles, Higgs/W/Z decays producing two, and $t \rightarrow W q$ decays producing three overlapping cones in the highly boosted regime. A follow-up study ${ }^{67}$ aims to identify boosted Higgs decays to pairs of b-hadrons.

\section{Outlook}

In this review, the many amazing manners in which AI and DL find applications in HEP and transform the ways data is simulated or analyzed are highlighted. By now the careful reader should be familial with acronyms like BDT, NN, ANN, MLP, DNN, CNN, RNN, LSTM, AE, IN, SGD and so on. These developments include all domains of physics. Building on the trend, the American Physical Society launched this year a new Topical Group on Data Science 68 (GDS).

A word of caution is in order: models developed and applied blindly as "black boxes" are good at learning correlations, not necessarily at helping to understand the underlying causations. Here physics is in an excellent position to do more by combining AI and ML with detailed domain knowledge. The symbiosis between the two fields is already producing encouraging results, and holds great promise for the future. The wide adoption of machine and deep learning in the physical sciences requires a cultural transformation and careful strategy, combining long term goals with step-by-step solving of current problems using the available data and human expertise, and learning new skills in the process. The unprecedented challenges 
of data-intensive science can be met with the breath-taking developments of new artificial intelligence methods and tools.

\section{Acknowledgments}

The author thanks his CMS colleagues from the high energy physics group at the University of Florida for the stimulating working atmosphere. This work is supported through NSF Grant No. PHY-1624356.

\section{References}

1. iVDGL proposal, http://www.phys.ufl.edu/〜avery/ivdgl/itr2001/proposal_all.pdf

2. Y. LeCun, Y. Bengio and G. Hinton, Nature 521, 436 (2015). doi:10.1038/nature14539

3. A. Radovic et al., Nature 560, no. 7716, 41 (2018). doi:10.1038/s41586-018-0361-2

4. K. Albertsson et al., J. Phys. Conf. Ser. 1085, no. 2, 022008 (2018) doi:10.1088/17426596/1085/2/022008 arXiv:1807.02876 [physics.comp-ph]].

5. G. Carleo, I. Cirac, K. Cranmer, L. Daudet, M. Schuld, N. Tishby, L. Vogt-Maranto and L. Zdeborov, arXiv:1903.10563 [physics.comp-ph].

6. Workshop on Multivariate Analysis in HEP and Astrophysics, February 11, 2008, https://www.caltech.edu/campus-life-events/master-calendar/workshop-onmultivariate-analysis-in-hep-and-astrophysics-2

7. M. Cauchy, Comptes Rendus Hebd. Séances Acad. Sci. no. 25, 536 (1847).

8. D. P. Kingma and J. Ba, arXiv:1412.6980 [cs.LG].

9. S. Chatrchyan et al. [CMS Collaboration], Phys. Lett. B, 716, 30 (2012). doi:10.1016/j.physletb.2012.08.021 arXiv:1207.7235 [hep-ex]].

10. G. Aad et al. [ATLAS Collaboration], Phys. Lett. B, 716, 1 (2012). doi:10.1016/j.physletb.2012.08.020 arXiv:1207.7214 [hep-ex]].

11. A. Hoecker et al., arXiv:physics/0703039, 2007.

12. R. Brun and F. Rademakers, Proceedings AIHENP'96 Workshop, Lausanne, Sep. 1996, Nucl. Inst. and Meth. in Phys. Res. A, 389, $81-86$ (1997). See also [root .cern.ch/] (http://root.cern.ch/).

13. A. M. Sirunyan et al. [CMS Collaboration], Phys. Rev. Lett., 122 no.2, 021801 (2019). doi:10.1103/PhysRevLett.122.021801 arXiv:1807.06325 [hep-ex]].

14. D. Bourilkov et al., EPJ Web Conf. 214, 06002 (2019). DOI: 10.1051/epjconf/201921406002

15. ATLAS Collab., ATLAS-CONF-2019-028, CERN, July 23, 2019.

16. T. Chen and C. Guestrin, doi:10.1145/2939672.2939785 arXiv:1603.02754 [cs.LG].

17. CMS Collab., CMS PAS HIG-18-031, CERN, July 14, 2019.

18. I. J. Goodfellow et al., arXiv:1406.2661 [stat.ML].

19. CMS Collab., CMS PAS HIG-18-032, CERN, March 22, 2019.

20. CMS Collab., CMS PAS HIG-18-030, CERN, June 24, 2019.

21. F. Chollet et al., "Keras". https://keras.io, 2015.

22. C. Adam-Bourdarios et al., JMLR: Workshop and Conference Proceedings, 42, 19-55, 2015.

23. https://www.kaggle.com/c/higgs-boson/ .

24. CERN Open Data Portal, http://opendata.cern.ch/.

25. M. Andrews et al., EPJ Web Conf. 214, 06031 (2019). DOI: 10.1051/epjconf/201921406031

26. K. He, X. Zhang, S. Ren and J. Sun, 2016 IEEE Conference on Computer Vision and Pattern Recognition, 770 (2016). 
27. B. Liu et al., EPJ Web Conf. 214, 06033 (2019). DOI: 10.1051/epjconf/201921406033

28. T. Vuillaume et al., EPJ Web Conf. 214, 06020 (2019). DOI: 10.1051/epjconf $/ 201921406020$

29. M. Kiehn et al., EPJ Web Conf. 214, 06037 (2019). DOI: 10.1051/epjconf/201921406037

30. https://www.kaggle.com/c/trackml-particle-identification/overview .

31. https://sites.google.com/site/trackmlparticle/ .

32. D. Derkach, M. Hushchyn and N. Kazeev, EPJ Web Conf. 214, 06011 (2019). DOI: 10.1051/epjconf/201921406011

33. F. Abudinn, EPJ Web Conf. 214, 06032 (2019). DOI: 10.1051/epjconf/201921406032

34. A. Sirunyan et al. [CMS Collaboration], JINST 13, no. 05, P05011 (2018) doi:10.1088/1748-0221/13/05/P05011 arXiv:1712.07158 [physics.ins-det]].

35. M. Abadi et al., TensorFlow: Large-scale machine learning on heterogeneous systems, 2015. http://tensorflow.org/, Software available from tensorflow.org.

36. M. Verzetti, EPJ Web Conf. 214, 06010 (2019). DOI: 10.1051/epjconf/201921406010

37. D. Bourilkov, EPJ Web Conf. 214, 06022 (2019). DOI: 10.1051/epjconf/201921406022

38. J. C. Collins and D. E. Soper, Phys. Rev. D 16, 2219 (1977).

39. T. Sjöstrand, S. Mrenna and P. Skands, JHEP 0605, 026 (2006).

40. T. Sjöstrand, S. Mrenna and P. Skands, Comput. Phys. Comm. 178, 852 (2008).

41. T. Sjöstrand et al., Comput.Phys.Commun. 191, 159-177 (2015).

42. I. Goodfellow et al., Proceedings of the International Conference on Neural Information Processing Systems, 26722680 (NIPS 2014).

43. L. de Oliveira, M. Paganini and B. Nachman, Comput. Softw. Big Sci. 1, no. 1, 4 (2017) doi:10.1007/s41781-017-0004-6 [arXiv:1701.05927 [stat.ML]].

44. M. Paganini, L. de Oliveira and B. Nachman, Phys. Rev. Lett. 120, no. 4, 042003 (2018) doi:10.1103/PhysRevLett.120.042003 arXiv:1705.02355 [hep-ex]].

45. K. Deja, T. Trzcinski and . Graczykowski EPJ Web Conf. 214, 06003 (2019). DOI: 10.1051/epjconf/201921406003

46. A. Pol et al., EPJ Web Conf. 214, 06008 (2019). DOI: 10.1051/epjconf/201921406008

47. K. Rinnert and M. Cristoforetti, EPJ Web Conf. 214, 06038 (2019). DOI: 10.1051/epjconf/201921406038

48. J. Brehmer, K. Cranmer, G. Louppe and J. Pavez, Phys. Rev. Lett. 121, no. 11, 111801 (2018) doi:10.1103/PhysRevLett.121.111801 arXiv:1805.00013 [hep-ph]].

49. A. De Simone and T. Jacques, Eur. Phys. J. C 79 , no. 4, 289 (2019) doi:10.1140/epjc/s10052-019-6787-3 arXiv:1807.06038 [hep-ph]].

50. S. Kullback, R. Leibler, Annals of Mathematical Statistics 22 (1), 7986 (1951). doi:10.1214/aoms/1177729694.

51. R. T. D'Agnolo and A. Wulzer, Phys. Rev. D 99, no. 1, 015014 (2019) doi:10.1103/PhysRevD.99.015014 arXiv:1806.02350 [hep-ph]].

52. G. Cybenko, Mathematics of Control, Signals, and Systems 2(4), 303314 (1989). doi:10.1007/BF02551274

53. R. D. Ball et al. [NNPDF Collaboration], JHEP 1504, 040 (2015). doi:10.1007/JHEP04(2015)040 arXiv:1410.8849 [hep-ph]].

54. R. D. Ball et al. [NNPDF Collaboration], Eur. Phys. J. C 77, no. 10, 663 (2017). doi:10.1140/epjc/s10052-017-5199-5 arXiv:1706.00428 [hep-ph]].

55. D. Bourilkov, hep-ph/0305126.

56. M. R. Whalley, D. Bourilkov and R. C. Group, hep-ph/0508110.

57. D. Bourilkov, R. C. Group and M. R. Whalley, hep-ph/0605240.

58. A. Buckley et al., Eur. Phys. J. C 75, 132 (2015). doi:10.1140/epjc/s10052-015-3318-8 arXiv:1412.7420 [hep-ph]]. 
59. J.-R. Vlimant et al., EPJ Web Conf. 214, 06025 (2019). DOI: 10.1051/epjconf/201921406025

60. V. Estrade et al., EPJ Web Conf. 214, 06024 (2019). DOI: 10.1051/epjconf/201921406024

61. A. Tsaris et al., J. Phys. Conf. Ser. 1085, 042023 (2018).

62. N. Tishby and N. Zaslavsky, In Information Theory Workshop (ITW), 2015 IEEE, $15,(2015)$.

63. R. Shwartz-Ziv and N. Tishby, arXiv:1703.00810 [cs.LG].

64. M. C. N. Cheng et al., arXiv:1906.02481 [cs.LG].

65. P. W. Battaglia, R. Pascanu, M. Lai, D. Rezende and K. Kavukcuoglu, arXiv:1612.00222 [cs.AI].

66. E. A. Moreno et al., arXiv:1908.05318 [hep-ex].

67. E. A. Moreno et al., arXiv:1909.12285 [hep-ex].

68. APS Topical Group on Data Science, https://www.aps.org/units/gds/index.cfm. 